\title{
Use of over-the-scope clips in the colon in clinical practice: results from a German administrative database
}

\section{다 (i)}

\section{Authors}

Dirk Horenkamp-Sonntag ${ }^{1}$, Judith Liebentraut ${ }^{1}$, Susanne Engel ${ }^{1}$, Christoph Skupnik ${ }^{1}$, David Albers ${ }^{2}$, Brigitte Schumacher ${ }^{2}$, Herbert Koop ${ }^{3}$

Institutions

1 Techniker Krankenkasse, Hamburg, Germany

2 Department of Internal Medicine and Gastroenterology, Elisabeth-Krankenhaus Academic Hospital, University of Duisburg-Essen, Essen, Germany

3 Formerly Department of General Internal Medicine and Gastroenterology, Helios Klinikum Berlin-Buch, Academic Hospital, Berlin, Germany

submitted 28.11.2019

accepted after revision 28.5.2020

published online 26.10 .2020

Bibliography

Endoscopy 2020; 52: 1103-1110

DOI 10.1055/a-1206-0778

ISSN $0013-726 \mathrm{X}$

(c) 2020. The Author(s).

This is an open access article published by Thieme under the terms of the Creative Commons Attribution-NonDerivative-NonCommercial License, permitting copying and reproduction so long as the original work is given appropriate credit. Contents may not be used for commecial purposes, or adapted, remixed, transformed or built upon. (https://creativecommons.org/licenses/by-nc-nd/4.0/)

Corresponding author

Herbert Koop, MD, Tölzer Str. 20, 14199 Berlin, Germany

Fax: +49-30-4012522

prof.koop@t-online.de

Supplementary material

Online content viewable at:

https://doi.org/10.1055/a-1206-0778
丹 Scan this QR-Code for the author commentary.

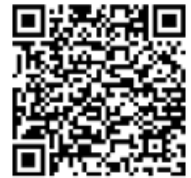

\section{ABSTRACT}

Background The efficacy and safety of over-the-scope (OTS) clips in the colon is limited. This study aimed to evaluate OTS clip use in the colon in routine colonoscopy.

Methods Using administrative data from a large health insurance company, patients with OTS clip placement during colonoscopy were identified and analyzed by specific administrative codes. Indication for OTS clipping was analyzed, and follow-up was evaluated for surgical and repeat endoscopic interventions.

Results In 505 patients, indications for OTS clips were iatrogenic perforations ( $n=80$; Group $A)$, polypectomy $(n=$ 315; Group B), colonic bleeding ( $n=51$; Group $C)$, and various underlying diseases ( $n=59$; Group D). In 11 Group A patients (13.8\%), surgical interventions occurred, mostly within 24 hours after clipping $(n=9)$, predominantly overstitching $(n=8)$. OTS clipping during polypectomy (Group B) was for complications (e. g. bleeding in $27 \%$ ) or was applied prophylactically. Only five patients required early surgery, three of whom had colorectal cancer. In four Group C patients $(7.8 \%)$, surgical resections were performed (persistent bleeding $n=1$, colorectal cancer $n=2$ ), while six patients underwent early repeat colonoscopy for recurrent bleeding. During further follow-up (days 11-30), 17 patients underwent resection for colonic neoplasms $(n=12)$ or persistent bleeding $(n=4)$, but only one case could be directly traced back to local OTS clip complication.

Conclusion Colonic OTS clipping appears safe and effective in selected indications and complications in clinical routine but must be anatomically and technically feasible, avoiding overuse. 

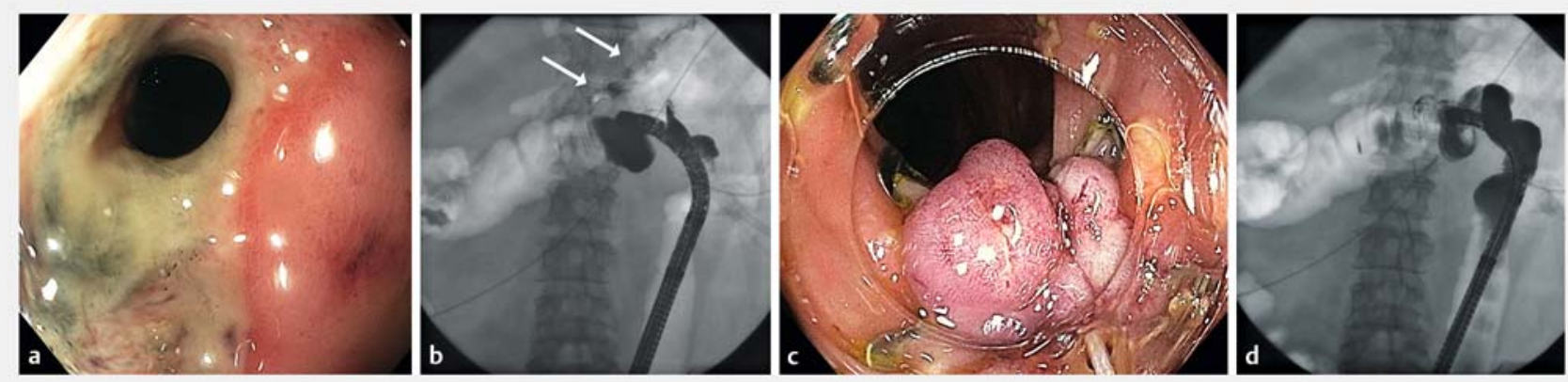

- Fig. 1 Application of the over-the-scope (OTS) clipping system in the colon. Example of fistula formation in the transverse colon after complicated gastric resection. a Endoscopic view of the fistula. b Fluoroscopy after administration of contrast agent to the colon, showing the fistula (arrows). c Endoscopic view after application of an OTS clip. $\mathbf{d}$ Confirmation of fistula sealing following administration of contrast medium (the OTS clip is visible proximal to the endoscope).

\section{Introduction}

Through-the-scope (TTS) clips have been introduced successfully into interventional endoscopic therapy, for example in cases with colonic (mostly iatrogenic) perforations [1] and colonic bleeding [2]; however, the clinical feasibility of closing perforations has been limited to small defects of less than 2 $\mathrm{cm}$. Recently, an over-the-scope (OTS) clip was developed primarily for the closure of endoscopic procedures such as fullthickness resection (EFTR) and natural orifice transluminal endoscopic surgery (NOTES) [3]. The OTS clip, also termed bear claw clip, is a large endoscopic clipping device made from nitinol. OTS clips have since also been used in the event of bleeding and perforations, predominantly in the upper gastrointestinal tract, with higher efficacy than TTS clips [4]. Data on OTS clip placement in the colon, however, are scarce. Although therapy has been reported to be very successful overall [5-11], most series have included only small numbers of patients and were performed in highly trained centers; thus, data are likely to be biased [12] and may therefore not represent the general effectiveness in clinical reality. Furthermore, the safety of OTS clip application, especially in the colon, has not been studied in large cohorts [13].

In the present study, a novel approach was chosen to investigate the safety of OTS clip placement in the colon. As application of OTS clips generates a specific administrative code in the German diagnosis-related group (DRG) reimbursement system, patients treated with OTS clip placement in the colon could be retrieved from a large database of a health insurance company. This enabled us to generate data representing routine clinical practice in colonoscopy in terms of indications, effectiveness, and complications of OTS clip use in the colon. Procedural application of OTS clips is exemplified by a case with a colonic fistula in $>$ Fig. 1.

\section{Methods}

Using a health insurance database of approximately 10 million insured patients, colonoscopy cases involving application of the OTS clipping system (Ovesco Endoscopy GmbH, Tübingen,
Germany) on the same day between January 2015 and March 2019 were identified. This database contains all procedures performed in hospitals as well as codes for outpatient procedures (International Classification of Diseases [ICD] codes10th revision; German operation and procedure codes [OPS] equivalent to International Classification of Procedures in Medicine [ICPM] codes; Einheitlicher Bewertungsmaßstab [EBM]) $[14,15]$. Codes for colonoscopy (OPS codes 16500, 16501, or 16502; EBM 01741, 13421, or 13422) and the specific OPS code for OTS clips (5469s3), medically documented within the scope of patient billing, were used for operationalization in the study. Patients undergoing EFTR were not included in the study as OTS clip use associated with the EFTR system generates a different OPS code. However, the code for OTS clip use does not differentiate between clipping in the small bowel and colon; therefore, all patients who underwent an upper Gl endoscopy on the same day of clipping were excluded from the analysis.

Using additional codes during the hospital stay (ICD and OPS), the presumed indication for OTS clipping was identified. Details for group allocation are shown in Table $1 \mathrm{~s}$ in the onlineonly supplementary material. Accordingly, the following groups were formed by a stepwise analysis ( $>$ Fig. 2): Group A, OTS clip closure of iatrogenic perforations occurring during colonoscopy based on ICD codes for wall injuries or peritonitis; Group B, OTS clip application in the context of polypectomy, also based upon respective OPS codes; Group C, colonic bleeding identified by specific ICD codes of colonic bleeding, acute anemia, and/or OPS codes for transfusion requirements. Group $D$ included a large variety of indications for use of OTS clips (e. g. fistulas, anastomotic leaks). Group D also included clip application at various times during an often prolonged hospital stay, and with very few endoscopic and/or surgical interventions occurring within the 10-day observation period; therefore, this group was omitted from further analysis.

If cases presented with codes potentially allocating patients to more than one group, we used a predefined hierarchy ( $\triangleright$ Fig. 2). Perforation was always placed in the primary position; for example, if a polypectomy led to perforation (and the perforation was coded), this patient would be assigned to 
Colonoscopy + same day OTS clip $(n=833)$

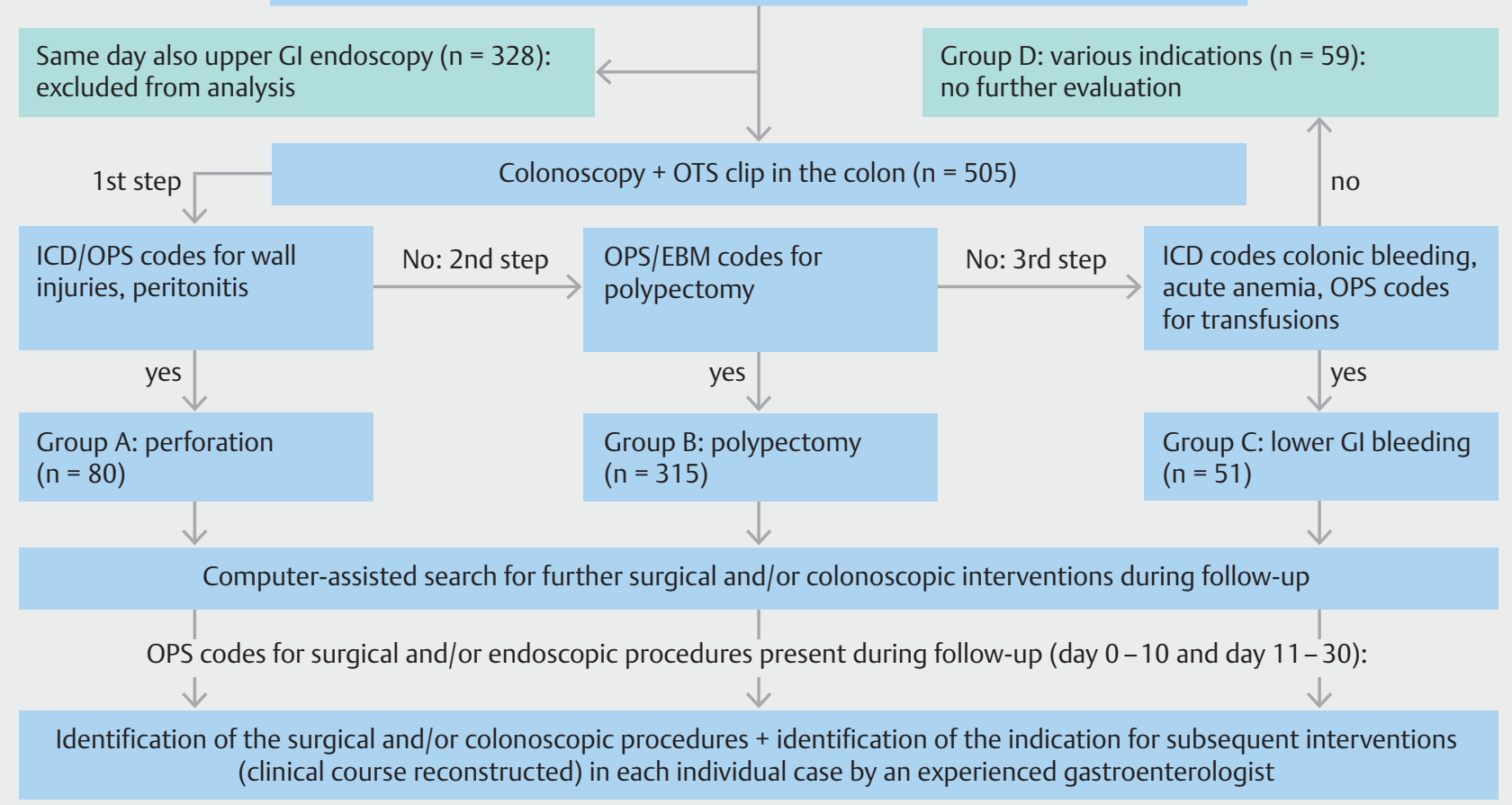

- Fig. 2 Analysis of data after over-the-scope clip application. OTS, over-the-scope; GI, gastrointestinal; ICD, International Classification of Diseases; OPS, operation and procedure codes; EBM, Einheitlicher Bewertungsmaßstab.

group A “perforation”. Bleeding due to polyp removal would primarily be assigned to "polypectomy" (Group B), not to "bleeding" (Group C). Consequently, therapeutic application of an OTS clip (e.g. due to perforation and/or bleeding) could only be identified if these events were documented by the specific codes.

The date of OTS clip application is exactly documented in claims data during the course of the hospital stay. Therefore, it is possible to track precisely further interventions occurring during follow-up. The search for such events was carried out along a list of predefined specific codes (OPS and EBM codes; Table 2s) to identify surgical procedures as well as colonoscopic reinterventions during follow-up. If any of these codes showed up during the computer-assisted search for events, every individual case was subsequently analyzed by a clinician experienced in both gastroenterology and endoscopy as well as in coding. This approach was necessary for the reconstruction of the individual clinical course, as the analysis of additional OPS and EBM codes alone did not, per se, give a clear picture of what had been happening to the patient during follow-up. Thus, information on the type of operative procedure and/or additional colonoscopic interventions (e.g. hemostatic techniques, additional polypectomy) that had been performed could be retrieved from the database. As data were available on the preceding medical records in each individual case, it was possible to differentiate between new diagnoses and preexisting disease (e. g. in the case of colorectal cancer [CRC]). Fi- nally, the results from individual evaluations were once again verified using computer-assisted tools.

In order to characterize the multimorbidity of patients, the Elixhauser score, a modification of the Charlson comorbidity index suitable for analyses with administrative data, was calculated for each patient. The Elixhauser score is a validated comorbidity measure, based on 30 selected ICD codes of comorbid diseases (e.g. heart diseases, diabetes, stroke) [16,17].

\section{Results}

During the study period, 833 patients underwent colonoscopy with simultaneous OTS clip application. A total of 328 patients also underwent upper $\mathrm{Gl}$ endoscopy on the same day, and these cases were excluded from further analysis, leaving 505 patients with an OTS clip solely in the colon for further evaluation ( $\triangleright$ Fig. 2). OTS clips were used with increasing frequency between 2015 and 2018, both in terms of the number of patients treated and the number of hospitals employing the OTS clipping system ( $>$ Fig. 3 ). Overall, the OTS clipping system was applied in 212 different hospitals with a wide range: clips were used only once during the study period in 120 hospitals, whereas one hospital employed OTS clips in 30 patients. The proportion of patients allocated to groups A-D (see below) remained relatively stable during this period.

Demographic details of patients are shown in $>$ Table 1 . In patients with colonic bleeding (Group C), there was a tendency 

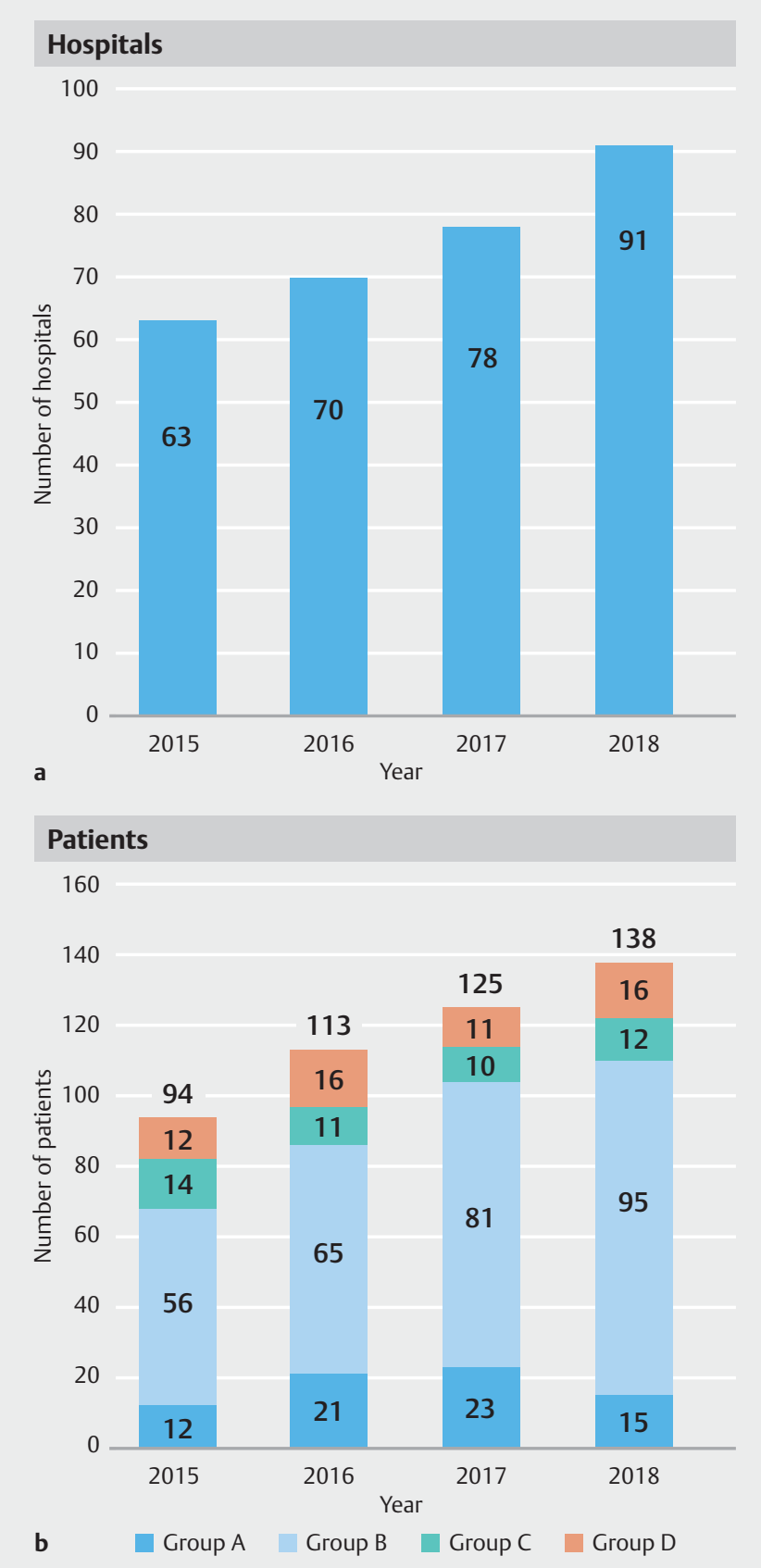

- Fig. 3 Use of the over-the-scope (OTS) clipping system between 2015 and 2018. a Number of hospitals using the device. b Number of patients treated by OTS clips, including indications for clip application. Total number of patients per year is shown above each column. Group A= perforations; Group B= polypectomy; Group C= bleeding; Group $D=$ others (e. g. fistulas, anastomotic leaks). toward more comorbidities compared with patients in the polypectomy group (Group B), as shown by a higher Elixhauser score, whereas the Elixhauser score was not different between Groups A and B.

\section{Group A: perforations}

OTS clips were applied in 80 patients for attempted closure of iatrogenic perforations that occurred during colonoscopy, $43.8 \%$ and $48.8 \%$ of which were also associated with polypectomy and bleeding, respectively. In 11 patients (13.8\%), coding included perforation, polypectomy, and bleeding.

In patients treated with an OTS clip for documented perforation, 11 patients (13.8\%) underwent an operative procedure during the short follow-up period of 10 days after clipping. In nine of these cases, surgery was carried out within approximately 24 hours after clipping. In eight patients, laparoscopic suturing of the defect was the only operative procedure, whereas in three patients (one of them with CRC) surgery included resection ( $\downarrow$ Table 2 ). Laparoscopic suturing might have been performed prophylactically or due to insufficient closure of the leak. Two patients underwent resection of the rightsided colon within 24 hours without evidence of CRC, and these cases probably represent early definitive treatment failure of leak closure by OTS clip placement.

Additional endoscopic procedures beyond the index colonoscopy and clipping ( $>$ Table 3 ) were carried out in five patients within the 10-day interval but colonoscopy did not include any further intervention in any of these patients. One death occurred in this group 1 day after OTS clipping in an 89year-old man with severe concomitant cardiac diseases.

\section{Group B: polypectomy}

The predominant indication for OTS clip application occurred in the context of polypectomy ( $n=315)$, with $27.0 \%$ of clip placements being associated with bleeding. In the remaining patients, it was not possible to confirm whether OTS clips were applied prophylactically or for treatment of complications (e. g. perforation and/or bleeding) because specific codes were missing from the database. Early colonic surgery within 10 days after clipping was necessary in five patients ( Table 2 ). Surgical interventions included resection in three cases (all of which had (RC), laparoscopic suturing in one case, and laparoscopy without any further procedure in one patient.

Endoscopic reinterventions early after OTS clip placement were performed in 17 patients (5.4\%) and included a variety of additional interventions targeted to hemostasis, additional polypectomy, or (presumed) inspection of the polypectomy site ( $>$ Table 3). No death occurred in this group during the 10-day follow-up.

\section{Group C: bleeding}

Colonic bleeding was another common indication for OTS clip application in the colon $(n=51)$ in addition to bleeding events in groups $\mathrm{A}$ and $\mathrm{B}$. One patient probably with an ongoing diverticular bleed (as exemplified by prior repeat attempts to stop bleeding endoscopically) underwent resection of the sigmoid 
- Table 1 Demographic data of patients $(n=505)$.

\begin{tabular}{|c|c|c|c|c|}
\hline Parameter & Group A (Perforation) & Group B (Polypectomy) & Group C (Bleeding) & Group D (Other) \\
\hline Patients, n (\%) & $80(15.8)$ & $315(62.4)$ & $51(10.1)$ & $59(11.7)$ \\
\hline Age, mean, years & 64 & 67 & 71 & 63 \\
\hline Males, \% & 51 & 62 & 67 & 61 \\
\hline Parallel coding of polypectomy, $n$ (\%) & $35(43.8)$ & NA & NA & ND \\
\hline Parallel coding of bleeding, $n$ (\%) & $39(48.8)$ & $85(27.0)$ & NA & ND \\
\hline Elixhauser score ( $95 \% \mathrm{Cl})$ & $7.0(4.8-9.1)$ & $6.1(5.1-7.0)$ & $9.6(6.8-12.3)$ & $8.4(5.9-10.8)$ \\
\hline Death $\leq 10$ days, $\mathrm{n}(\%)$ & $1(1.3)$ & 0 & $1(2.0)$ & 0 \\
\hline Surgery $\leq 10$ days, $\mathrm{n}(\%)$ & $11(13.8)$ & $5(1.6)$ & $4(7.8)$ & 0 \\
\hline Repeat colonoscopy $\leq 10$ days, $\mathrm{n}$ (\%) & $5(6.3)$ & $17(5.4)$ & $6(11.8)$ & $1(1.7)$ \\
\hline
\end{tabular}

- Table 2 Surgical interventions within 10 days after application of over-the-scope clips.

\begin{tabular}{|l|l|l|l|}
\hline Surgical intervention & $\begin{array}{l}\text { Group A (Perforation) } \\
(\mathbf{n = 1 1 )}\end{array}$ & $\begin{array}{l}\text { Group B (Polypectomy) } \\
(\mathbf{n = 5})\end{array}$ & $\begin{array}{l}\text { Group C (Bleeding) } \\
\text { (n=4) }\end{array}$ \\
\hline Laparoscopy only & 0 & 1 & 0 \\
\hline Laparoscopic suturing & 8 & 1 & 0 \\
\hline - Combined with stoma and lavage & 1 & 0 & - \\
\hline Resection & 3 & 3 & 3 \\
\hline - Patients with CRC & 1 & 3 & 2 \\
\hline Surgery unrelated to indication for OTS clips & 0 & 0 & 1 \\
\hline CRC, colorectal cancer; OTS, over the scope. & & & \\
\hline
\end{tabular}

- Table 3 Colonoscopic reinterventions within 10 days after application of an over-the-scope clip.

\begin{tabular}{|l|l|l|l|}
\hline Endoscopic intervention & $\begin{array}{l}\text { Group A (Perforation) } \\
(\mathbf{n = 5}\end{array}$ & $\begin{array}{l}\text { Group B (Polypectomy) } \\
\text { (n=17) }\end{array}$ & $\begin{array}{l}\text { Group C (Bleeding) } \\
(\mathbf{n = 6})\end{array}$ \\
\hline Colonoscopy only & 5 & 6 & 1 \\
\hline Colonoscopy+ additional polypectomy & 0 & 3 & 0 \\
\hline Colonoscopy+ clip/injection/thermal/hemostatic agents & 0 & 6 & 3 \\
\hline Colonoscopy+ new OTS clip & 0 & 2 & 2 \\
\hline After removal of first OTS clip & - & 1 & 0 \\
\hline OTS, over-the-scope. & & & \\
\hline
\end{tabular}

colon 8 days after OTS clip application, whereas in two cases, resection was associated with CRC.

Repeat colonoscopy was performed in six patients early after OTS clip application; in five of these cases, repeat interventions were again probably aimed at controlling bleeding as exemplified by interventions using TTS clips, injections, and even a second OTS clip in two cases. The estimated overall rebleeding rate (as identified by repeat endoscopic intervention or surgery during follow-up, excluding cases with (RC) within 10 days after
OTS clipping was $10.2 \%$. One death occurred in an 88 -year-old woman with CRC and severe heart failure 3 days after OTS clip placement.

\section{Safety}

In order to capture all delayed complications beyond the 10day period, surgical procedures were analyzed in groups $\mathrm{A}, \mathrm{B}$, and $C$ up to 30 days after OTS clip placement. As shown in - Table 4, 17 patients underwent surgery 11-30 days after 
- Table 4 Surgical interventions between days 11 and 30 after application of over-the-scope clips.

\begin{tabular}{|l|l|l|l|}
\hline Surgical intervention and indication & $\begin{array}{l}\text { Group A (Perforation) } \\
(\mathbf{n = 6 )}\end{array}$ & $\begin{array}{l}\text { Group B (Polypectomy) } \\
(\mathbf{n = 8 )}\end{array}$ & $\begin{array}{l}\text { Group C (Bleeding) } \\
(\mathbf{n = 3})\end{array}$ \\
\hline Resection & 5 & 8 & 2 \\
\hline Resection with stoma & 1 & - & 1 \\
\hline Cause for surgery & & & \\
\hline - CRC & 3 & 6 & 2 \\
\hline - Adenoma & - & 1 & - \\
\hline - Bleeding & 2 & 1 & 1 \\
\hline - Unknown & 1 & - & - \\
\hline CRC, colorectal cancer. & & & \\
\hline
\end{tabular}

OTS clip placement (mean 20 days). Surgery was associated with CRC $(n=11)$ and colon adenoma $(n=1)$. In four patients, resection was carried out most likely for persistent or recurrent bleeding, as revealed by preceding endoscopic interventions using hemostatic tools. In one Group A patient, a plausible cause for surgery could not be identified and might represent delayed failure of the OTS clip to permanently close the perforation.

\section{Discussion}

Indication, effectiveness, and safety of OTS clip placement in the colon is not yet established. OTS clips were primarily developed for NOTES and were subsequently [18] used for EFTR (not included in this study); however, reports of colonic OTS clip application describe only small case numbers mainly from highly specialized centers [5-11] or may be prone to publication bias [19], and may not reflect routine clinical use. Therefore, the clinical efficacy of OTS clip placement in the colon still needs further evaluation. As use of OTS clips generates a specific administrative code in the German DRG system, which is relevant for reimbursement, analysis of administrative data offers great potential to study the clinical efficacy of endoscopic innovations in clinical practice. Secondary data from the statutory health insurance funds may be used for the measurement of outcomes over long periods of time [14]. Even failures are more likely to be coded, as the specific code leads to substantial increases in the reimbursement. The database used in the current study derives from the largest health insurance company in Germany (Techniker Krankenkasse), with about 10 million insured patients. Although not fully representative of all patients in Germany, results are likely to give a realistic image of the German health system. Thus, data obtained on the use of the OTS clipping system in this study are very likely to reflect clinical routine conditions.

In the present study, we identified 505 patients who received an OTS clip in the colon. The OTS clips were not extensively used, and closure of perforations must have been performed in many instances without extensive experience in using this clipping system. Concomitant coding (ICD-10, OPS [ICPM-like] codes) enabled us to identify the presumed indication for clip application in a large number of patients.

A total of 80 patients experienced a perforation, either during colonoscopy alone or in combination with a polypectomy and/or bleeding; these patients were subsequently treated with an OTS clip in this emergency situation. This is by far the largest reported cohort of patients with colonic perforations treated with an OTS clip. It is acknowledged that patients are only suitable for clipping if the perforation is recognized immediately during the endoscopic procedure, and the OTS clip placement must be technically feasible too. This cohort admittedly represents a subset of patients with a more favorable prognosis even if they require surgery [20]. However, the rate of additional surgical interventions after OTS clipping was remarkably low, occurring in only $11 / 80$ patients. Furthermore, the majority of patients were treated solely by laparoscopic suturing, and only three patients required resection (one case with (RC). It was not possible to evaluate whether laparoscopic suturing was performed for unsuccessful clipping or in addition to OTS clipping to secure the closure. Even if all patients who underwent subsequent surgery are regarded as treatment failures, OTS clip placement presumably replaced surgery in more than $85 \%$ of patients. The success rate is thus similar to a report by Weiland at al. [19].

Closure of iatrogenic perforations by OTS clip placement requires the same watchful observation as surgery to ensure that complications are not missed: in principle, the same delayed complications may occur after application of the OTS clip system as after surgery, and this may have been the case in two patients in the cohort of patients in this study. However, even in patients with primary failure of the closure by clipping, secondary clipping is an option, potentially avoiding surgery. Thus, the advantage of OTS clipping may evolve as an alternative to surgery, and even in cases of incomplete closure necessitating surgery, contamination of the peritoneal cavity and peritonitis may be reduced.

Polypectomy was the dominant indication for use of OTS clips. For methodological reasons, it was possible to only partially identify the clinical situation that led to clipping. Bleeding was associated with OTS clipping in $27.0 \%$ of patients. In the re- 
maining patients, OTS clip application was most likely also performed due to temporary bleeding and eventually in a few patients with (presumed) perforation during polypectomy; however, respective coding is lacking. Only five patients required surgery $(1.6 \%)$, which was due to CRC in three patients; the rate of endoscopic reinterventions (5.4\%) was low. These findings suggest that OTS clipping contributed substantially to a definitive therapy for polypectomy complications; moreover, it is likely that large polyps were removed and required OTS clips afterwards as the data were derived from hospitals only. Recently, TTS clip application following polypectomy has been shown to reduce the risk of bleeding [21,22]; however, the OTS clips are supposed to be even more effective, as demonstrated in recurrent peptic ulcer bleeding [23]. This hypothesis requires testing in further studies.

Colonic bleeding is an endoscopic challenge, as this condition is associated with multimorbidity and use of anticoagulants [24]. Therefore, this cohort has a different prognosis from patients with bleeding after polypectomy. Identification of the exact bleeding source using administrative data is difficult, as specific ICD codes exist only for selected types of colonic bleeding (e.g. diverticular bleeding). Though lower gastrointestinal (particularly diverticular) bleeding stops spontaneously in $80 \%-85 \%$ of cases [25], endoscopic efforts to stop an active bleed are frequently unsuccessful in the colon. Despite progress made by TTS clips, rebleeding remains a problem [26]. Identification of the exact bleeding source is necessary for precise clip placement, yet often difficult during colonoscopy. A clip that grasps more tissue may therefore be advantageous [27]. The present study seems to support this hypothesis, as only one patient underwent surgery (resection of the sigmoid colon) probably due to ineffective hemostasis, whereas the remaining patients clearly required surgery for bleeding CRC. The estimated rebleeding rate (excluding cancer patients) of $10 \%$ seems lower compared with $16 \%$ reported in a recent metaanalysis [28]. Endoscopists should, however, always critically evaluate whether the indication for an OTS clip is appropriate and should definitively avoid overuse.

There has been some concern about the long-term safety of OTS clips. Complications reported to date [29, 30] include those that require surgery not necessarily within 10 days of clip placement. Therefore, surgical procedures beyond the 10day interval were also analyzed in the present study to identify delayed failures and complications that might result from altered local blood perfusion. However, up to 30 days after OTS clipping, surgery was performed almost exclusively for underlying diseases (CRC, bleeding, etc.), and in only one case out of 505 patients may thus be classified as delayed failure. This matches reports related to OTS clip-related failures and complications; however, watchful observation is mandatory in patients after OTS clip placement even though it is possible to replace an existing OTS clip [31], which was also done in the present cohort.

Data for statutory health insurance are primarily collected for billing of medical benefits and can therefore only be used as secondary data for scientific analyses in healthcare research. In order to be able to use this huge analytic potential of claims data on a valid basis, limitations of secondary data must be taken into consideration $[14,15]$. In particular, some ICD codes lack some degree of diagnostic accuracy; for example, ICD codes for lower gastrointestinal bleeding may lack precise definitions. Whereas a time-based assignment is exactly feasible by means of OPS codes, the exact date of ICD events is not always determinable.

To combine day-based OPS information with period-related ICD diagnoses optimally, clinical (medical) expertise was systematically interlinked with data analytical competence. The resultant knowledge enabled a more complex and also a more detailed operationalization. Subsequently, more precise classification of patients into the different treatment groups and improved recording of complications could be ensured.

The strengths of this study include the novel approach to evaluating endoscopic innovations in a large number of patients, substantially exceeding previously reported cohorts in number. Data were obtained from routine clinical practice with minimal risk of under-reporting. Study limitations include the fact that the analysis was based on a registry consisting of administrative data; codes for diagnostic and therapeutic procedures and diagnoses had to be translated back into the clinical course without access to clinical reports. Although one of the authors with profound clinical experience looked at many individual cases (e.g. all patients with repeat endoscopic and/or surgical interventions), reconstruction of the clinical course could not be achieved in every single case, though it was probably achieved in the vast majority of patients.

In conclusion, the application of OTS clips in the colon offers a new tool for various clinical settings. The OTS clipping system as an alternative to surgical procedures is restricted to use in suitable patients with iatrogenic perforations and may enhance the prophylactic and therapeutic armamentarium in difficult polypectomies and colonic bleeding; however, overuse should be strictly avoided.

Competing interests

The authors declare that they have no conflicts of interest.

\section{References}

[1] Boumitri C, Kumta NA, Patel M et al. Closing perforations and postperforation management in endoscopy: duodenal, biliary and colonic. Gastrointest Endosc Clin N Am 2015; 25: 47-54

[2] Ishii N, Omata F, Nagata N et al. Effectiveness of endoscopic treatments for colonic diverticular bleeding. Gastrointest Endosc 2018; 87: 58-66

[3] Weiland T, Fehlker M, Gottwald T et al. Performance of the OTSC System in the endoscopic closure of iatrogenic gastrointestinal perforations: a systematic review. Surg Endosc 2013; 27: 2258-2274

[4] Schmidt A, Gölder S, Goetz M et al. Over-the-scope clips are more effective than standard endoscopic therapy for patients with recurrent bleeding of peptic ulcer. Gastroenterology 2018; 155: 674-686

[5] Kirschniak A, Subotova N, Zieker D et al. The over-the-scope clip (OTSC) for the treatment of gastrointestinal bleeding, perforations and fistulas. Surg Endosc 2011; 25: 2901-2905 
[6] Voermans RP, le Moine O, von Renteln D et al. Efficacy of endoscopic closure of acute perforations of the gastrointestinal tract. Clin Gastroenterol Hepatol 2012; 10: 603-608

[7] Haito-Chavez Y, Law JK, Kratt T et al. International multicenter experience with an over-the-scope clipping device for endoscopic management of GI defects. Gastrointest Endosc 2014; 80: 610-622

[8] Mizrahi I, Eltavil R, Haim N et al. The clinical utility of over-the-scope clip for the treatment of gastrointestinal defects. J Gastrointest Surg 2016; 20: 1942-1949

[9] Gubler C, Bauerfeind P. Endoscopic closure of iatrogenic gastrointestinal tract perforations with the over-the-scope clip. Digestion 2012; 85: 302-307

[10] Mangiavillano B, Caruso A, Manta R et al. Over-the-scope clips in the treatment of gastrointestinal tract iatrogenic perforation: a multicenter retrospective study and a classification of gastrointestinal tract perforations. World J Gastroenterol 2016; 8: 315-320

[11] Richter-Schlag H], Glatz T, Walker $C$ et al. First-line treatment with over-the-scope clips significantly improves the primary failure and rebleeding rates in high-risk gastrointestinal bleeding: a single center experience with 100 cases. World J Gastroenterol 2016; 22: 91629171

[12] Bemelman WA, Baron TH. Endoscopic management of transmural defects, including leaks, perforations, and fistulas. Gastroenterology 2018; 154: 1938-1946

[13] Gralnek IM, Khamaysi Y. Is the over-the-scope clip device a first-line or rescue therapy for patients at high risk for gastrointestinal bleeding? Clin Gastroenterol Hepatol 2018; 16: 627-629

[14] Horenkamp-Sonntag D. Versorgungsforschung und Qualitätsmessung mit GKV-Routinedaten. Baden Baden: Nomos Verlag; 2017

[15] Horenkamp-Sonntag D, Linder R et al. Datenzugang und Datenvalidierung: Prüfung der Datenqualität und Validität von GKV-Routinedaten. In: Swart E, Ihle P, Gothe H, Matusiewicz D. Routinedaten im Gesundheitswesen. Handbuch Sekundärdatenanalyse: Grundlagen, Methoden und Perspektiven. 2nd edn. Bern: Huber; 2014: 314-330

[16] Fabbian F, de Giorgi A, Maietti E et al. A modified Elixhauser score for predicting in-hospital mortality in internal medicine admissions. Eur ] Intern Med 2017; 40: 37-42

[17] Austin SR, Wong YN, Uzzo RG et al. Why summary comorbidity measures such as the Charlson Comorbidity Index and Elixhauser score work. Med Care 2015; 53: e65-72
[18] Schmidt A, Benya T, Schumacher B et al. Colonoscopic full-thickness resection using an over-the-scope device: a prospective multicentre study in various indications. Gut 2018; 67: 1280-1289

[19] Weiland T, Rohrer S, Schmidt A et al. Efficacy of the OTSC system in the treatment of $\mathrm{Gl}$ bleeding and wall defects: a PMCF meta-analysis. Minim Invasive Ther Allied Technol 2020; 29: 121-139

[20] Iqbal CW, Chun YS, Farley DR. Colonic perforations: a retrospective review. J Gastrointest Surg 2005; 9: 1229-1236

[21] Pohl H, Grimm IS, Moyer MT et al. Clip closure prevents bleeding after resection of large colon polyps in a randomized trial. Gastroenterology 2019; 157: 977-984

[22] Zhang QS, Han B, Xu JH et al. Clip closure of defect after endoscopic resection in patients with larger colorectal tumors decreased the adverse events. Gastrointest Endosc 2015; 82: 904-909

[23] Schmidt A, Gölder S, Goetz M et al. Over-the-scope clips are more effective than standard endoscopic therapy for patients with recurrent bleeding of peptic ulcers. Gastroenterology 2018; 155: 674-686

[24] Oakland K, Guy R, Uberoi R et al. Acute lower GI bleeding in the UK: patient characteristics, interventions and outcomes in the first nationwide audit. Gut 2018; 67: 654-662

[25] Farrell JJ, Friedman LS. Review article: the management of lower gastrointestinal bleeding. Aliment Pharmacol Ther 2005; 21: 1281-1298

[26] Moss AJ, Tuffaha H, Malik A. Lower GI bleeding: a review of current management, controversies and advances. Int J Colorect Dis 2016; 31: 175-188

[27] Manta R, Mangiafico S, Zullo A et al. First-line endoscopic treatment with over-the-scope clips in patients with either upper or lower gastrointestinal bleeding: a multicenter study. Endosc Intern Open 2018; 06: E1317-E1321

[28] Kouanda AM, Somsouk M, Sewell JL et al. Urgent colonoscopy in patients with lower GI bleeding: a systematic review and meta-analysis. Gastrointest Endosc 2017; 86: 107-117

[29] Rodriguez Lopez M, Blanco Jl, Martinez R et al. Clipping for endoscopic perforation: initial success but late complication requiring surgery. Surg Endosc 2013; 37: 3948-3950

[30] Fischer A, Richter-Schrag HJ. Over-the-scope clip (OTSC)-induced colonic obstruction necessitating sigmoid resection. Endoscopy 2015; 47: E157-E158

[31] Caputo A, Schmidt A, Caca K et al. Efficacy and safety of the remove system for OTSC and FTRD clip removal: data from PMCF removal. Minim Invasive Ther Allied Technol 2018; 27: 138-142 